\title{
Periodontia versus Implantodontia: Uma breve revisão do dilema da extração
}

\section{dentária}

\author{
Periodontics versus Implantodontics: A short review of the dilemma of tooth extraction \\ Periodoncia versus implantodoncia: Una breve revisión del dilema de la extracción dental
}

Recebido: 01/03/2021 | Revisado: 06/03/2021 | Aceito: 09/06/2021 | Publicado: 23/06/2021

\author{
Fabio Anibal Goiris \\ ORCID: https://orcid.org/0000-0001-8721-4877 \\ Universidade Estadual de Ponta Grossa, Brasil \\ E-mail: fgoiris@hotmail.com \\ Priscila Smolareck \\ ORCID: https://orcid.org/0000-0001-7845-0261 \\ Universidade Estadual de Ponta Grossa, Brasil \\ E-mail: pcsmolarek@gmail.com \\ Juliana Larocca de Geus \\ ORCID: https://orcid.org/0000-0001-9633-0474 \\ Faculdade Paulo Picanço, Brasil \\ E-mail: juliana.degeus@facpp.edu.br
}

\begin{abstract}
Resumo
O tratamento de dentes acometidos por doenças periodontais tem se mostrado eficaz em mantê-los na cavidade oral ou como suporte para próteses fixas por longos períodos, apresentando uma sobrevida em torno de $90 \%$. Porém, no que se refere à inter-relação da Periodontia e da Implantodontia, pesquisas recentes têm mostrado que o número de exodontias de dentes danificados pela doença periodontal vem aumentando significativamente. Além da cárie e da doença periodontal, a ação do fórceps do dentista é uma das principais causas de exodontias, que é utilizada nas mais diversas situações e geralmente seguindo critérios próprios dos profissionais. $\mathrm{O}$ avanço da Implantodontia e o surgimento das indústrias de implantes mudaram vários aspectos do prognóstico quanto à manutenção (ou não) dos dentes. A Periodontia é uma disciplina que busca desenvolver metodologias e decisões clínicas baseadas em evidências científicas e em prognósticos éticos que realmente favoreçam os pacientes. Este estudo visa contribuir para esse debate e apresenta casos clínicos em que dentes com lesão periodontal não foram encaminhados para extração e foram preservados e mantidos na cavidade oral.
\end{abstract}

Palavras-chave: Periodontia; Cirurgia bucal; Carga Imediata em implante dentário.

\begin{abstract}
The treatment of teeth affected by periodontal diseases has been shown efficient in keeping them in the oral cavity or as support for fixed prosthesis for long periods, showing around a $90 \%$ survival rate. However, regarding the interrelation of Periodontics and Implantology, recent research has shown that the number of extractions of teeth damaged by the periodontal disease has been increasing significantly. In addition to caries and periodontal disease, the dentists' forceps action is one of the main causes of extraction, which is used in the most varied situations and usually following the professionals' own criteria. The implantology advance and the appearance of implant industries changed several aspects of the prognosis regarding keeping the teeth (or not). Periodontics is discipline that seek to develop methodologies and clinical decisions based on scientific evidence and on ethics prognosis that really favor the patients. This study aims at contributing to this debate and presents clinical cases in which periodontal damaged teeth were not referred to extraction and were preserved and kept in the oral cavity.
\end{abstract}

Keywords: Periodontics; Oral surgery; Immediate dental implant loading.

\section{Resumen}

El tratamiento de los dientes afectados por enfermedades periodontales se ha mostrado eficaz para mantenerlos en la cavidad bucal o como soporte de prótesis fijas durante largos períodos, mostrando una tasa de supervivencia en torno al 90\%. Sin embargo, con respecto a la interrelación de Periodoncia e Implantología, investigaciones recientes han demostrado que el número de extracciones de dientes dañados por la enfermedad periodontal ha aumentado significativamente. Además de la caries y la enfermedad periodontal, la acción del fórceps del odontólogo es una de las principales causas de extracción, que se utiliza en las más variadas situaciones y habitualmente siguiendo los propios criterios de los profesionales. El avance de la implantología y la aparición de las industrias de implantes cambiaron varios aspectos del pronóstico en cuanto al mantenimiento de los dientes (o no). La periodoncia es una disciplina que busca desarrollar metodologías y decisiones clínicas basadas en la evidencia científica y en el 
pronóstico ético que realmente favorezca a los pacientes. Este estudio tiene como objetivo contribuir a este debate y presenta casos clínicos en los que los dientes con daño periodontal no fueron referidos a extracción y fueron preservados y conservados en la cavidad bucal.

Palabras clave: Periodoncia; Cirurga bucal; Carga inmediata del implante dental.

\section{Introdução}

A cárie dentária costumava ser o motivo mais comum para a extração dentária, enquanto a doença periodontal apareceu como o fator mais crítico na perda dentária, sendo a última predominante na faixa etária acima de 40 anos (Hull et al. 1997). No entanto, os dados clínicos mostram que na "Era dos Implantes", um grande aumento foi observado na extração de dentes danificados pela doença periodontal (Lindhe 2007). Um dos motivos da perda dentária, raramente discutido, é a ação do fórceps do dentista, utilizado nas mais diversas situações e geralmente seguindo indicações muito pessoais dos mesmos profissionais (Lundgren et al. 2008).

De acordo com Aida (2021), a perda dentária é a consequência final de comportamentos inadequados de saúde bucal e doenças bucais ao longo da vida. A perda de dentes deteriora substancialmente a qualidade de vida e a saúde geral. O fardo da perda dentária é maior nas condições bucais, causando uma perda de 7,6 milhões de anos de vida ajustados por incapacidade em todo o mundo. A condição socioeconômica e os fatores socioambientais, como abastecimento de água fluoretada, acessibilidade ao atendimento odontológico e capital social, afetam a perda dentária como fatores secundários. A prevenção de doenças bucais, mudanças nos comportamentos de saúde bucal e a melhoria do ambiente social podem reduzir o risco de perda dentária (Aida 2021).

O tratamento da doença periodontal está, portanto, em uma encruzilhada. Com o rápido avanço da Implantodontia e o surgimento da indústria de implantes, a decisão de manter os dentes naturais que foram danificados pela doença periodontal tem enfrentado o impacto de novos parâmetros de prognóstico. Não há dúvidas de que o tratamento com implantes tem apresentado vasta e sólida documentação científica, resultado de pesquisas (e prática clínica), cujos resultados são corroborados positivamente em todo o mundo (Lekholm et al. 1999, Lang et al. 2004, Pjetursson et al. 2004, Jnag et al. 2011, Levin et al. 2015, Schimmel et al. 2018, Tallarico et al. 2018).

Mais recentemente, as técnicas regenerativas em implantodontia foram incorporadas, como distração alveolar, regeneração óssea guiada (GBR) e enxerto ósseo (Fernandez-Medina et al. 2021). Porém, o avanço da implantodontia também trouxe alguns prognósticos duvidosos: dentes que receberiam tratamento periodontal para serem mantidos na boca passaram a ser encaminhados para extração. Portanto, o maior desafio para a Periodontia é desenvolver metodologias e decisões clínicas baseadas em a) evidências científicas, principalmente para prevenir a ocorrência de extrações desnecessárias (Riccuci \& Grosso 2006, Greenstein et al. 2008, Nemcovsky et al. 2017), b) valores bioéticos bem estabelecidos (Naik et al. 2016), e c) prognóstico que favorece os pacientes (Avila et al. 2009, Setzer \& Kim 2017, Sanz et al. 2020). Por isso, essa revisão de literatura tem como objetivo contribuir para esse debate.

\section{Metodologia}

O presente artigo se trata de uma revisão de literatura (Pereira et al. 2018) com relatos de casos clínicos relacionados ao tema. Foi realizada uma busca na literatura científica de artigos sobre a Periodontia e a Implantodontia e sua relação com a manutenção dental. Além disso, o artigo foi ilustrado com figuras de casos clínicos de pacientes que necessitaram de tratamento periodontal. Todos os pacientes que tiveram seus casos relatados assinaram um Termo de Consentimento Livre e Esclarecido e autorizaram o uso das imagens. 


\section{Casos e Discussão}

O alto número de extrações dentárias é causado por diversos fatores; muitas vezes, erroneamente referidas como 'causas naturais', tais como: a) perda da inserção clínica inicial do periodonto seguida de falta de tratamento; b) aumento do número de pessoas incluídas nos grupos de risco para doenças periodontais, como fumantes e diabéticos; e c) susceptibilidade, geralmente desconhecida, de certos pacientes à doença periodontal (Ong 1998).

Além disso, outro motivo para o aumento das extrações dentárias é o fato de os pacientes, em geral, terem a ideia (muitas vezes sugerida pelos dentistas) de que é melhor extrair dentes afetados pela doença periodontal e substituí-los por implantes. Alguns dizem que quanto mais tempo esses dentes permanecem na boca, maior se torna a perda óssea alveolar (Riccuci \& Grosso 2006). Quem afirma isso desconsidera e ignora o tratamento periodontal. Autores como Lindhe (2007) apontam que essa prática que desconsidera o tratamento periodontal tem resultado em um aumento de dentes extraídos que ainda podem ser tratados. Um conceito, na maioria das vezes impreciso e apressado, considera o prognóstico de dentes danificados periodontais terrivelmente ruim, enquanto o prognóstico do implante é considerado muito positivo. Essa inversão de lógica, como afirma Lindhe (2007), também pode afetar o ensino na Odontologia. Portanto, não parece correto pensar que todos os pacientes devam ser tratados da mesma forma com os implantes.

De acordo com Lundgren, Rylander e Laurell (2008) e Lang-Hua et al. (2014), há uma impressão geral, nem sempre correta, de que o tratamento com implantes apresenta maiores taxas de sucesso quando comparado ao complicado e demorado tratamento periodontal. Por esse motivo, cada vez mais dentes têm sido extraídos e substituídos por implantes em pacientes que apresentam doença periodontal (moderada a avançada) sem proporcionar tratamento adequado e, principalmente, sem verificar a susceptibilidade dos pacientes à progressão das doenças. Portanto, dentes que poderiam ser salvos são substituídos por implantes, muitas vezes com encaminhamentos duvidosos.

Existem duas tendências na relação interdisciplinar entre a Periodontia e a Implantodontia: aquela que defende a extração de dentes com prognóstico desfavorável e sua substituição por implantes. Outra que preconiza o uso de todo esforço profissional para preservar o periodonto remanescente e manter o dente em funcionamento. Segundo Lascala, Jardini e Melo (2003), os extremos entre essas duas tendências não são recomendados. Esses autores defendem a ideia de que os profissionais devem sempre manter os dentes naturais, respeitando o conhecimento científico existente (principalmente no que se refere aos avanços no tratamento periodontal), as características individuais dos pacientes e suas condições socioeconômicas.

O conhecimento adequado da Periodontia é essencial para encontrar caminhos específicos no prognóstico para prevenir medidas inconsequentes ou desnecessárias como a exodontia (Patel, 2020). Giannobile e Lang (2016) escreveram que uma tendência que afetou a prática clínica nas últimas duas décadas foi a ênfase reduzida em "salvar dentes comprometidos". $\mathrm{Na}$ verdade, estudos demonstraram que aqueles com menos treinamento em Periodontia e Implantodontia geralmente aplicam esforços reduzidos para lidar com a retenção dentária. Observa-se que indivíduos menos treinados costumam recomendar extração versus retenção do dente. Como tal, muitos dentes estão sendo condenados em estágios iniciais de perda de suporte ósseo devido ao expediente que se presta a livrar rapidamente um dente problemático e fornecer um novo implante de substituição de dente, deduzido como "mais novo, melhor".

De acordo com Lundgren, Rylander e Laurell (2008) e Lang-Hua et al. (2014), há uma impressão geral, nem sempre correta, de que o tratamento com implantes apresenta maiores taxas de sucesso quando comparado ao complicado e demorado tratamento periodontal. Por esse motivo, cada vez mais dentes têm sido extraídos e substituídos por implantes em pacientes que apresentam doença periodontal (moderada a avançada) sem proporcionar tratamento adequado e, principalmente, sem verificar a susceptibilidade dos pacientes à progressão das doenças. Portanto, dentes que poderiam ser salvos são substituídos por implantes, muitas vezes com encaminhamentos duvidosos.

A maioria dos sistemas de implantes são, como os dentes naturais, suscetíveis a complicações biológicas. Doering 
(2013), afirma que poucos estudos fornecem dados sobre a prevalência de doenças peri-implantares e, em 2000, apenas 26 artigos com a palavra-chave "peri-implantite" foram identificados por pesquisa no PUBMED. Esse número subiu para $192 \mathrm{em}$ 2012, sugerindo um maior interesse na área. De acordo com Giannobile, Lang, (2016) essas complicações peri-implantares resultam em opções extremamente difíceis de tratar, incluindo terapia mecânica local e antibióticos, cirurgia de ressecção, regeneração ou, em muitos casos, remoção. A crença errônea de que os implantes produzem um melhor prognóstico em longo prazo foi agora claramente rejeitada em vários estudos comparativos e revisões sistemáticas. Dentes comprometidos por doença periodontal ou problemas endodônticos podem ter uma longevidade que ultrapassa em muito a de um implante em média (Carnevale et al. 1998, Lang 2012, Salvi et al. 2014, Klinge et al. 2015).

No campo da Endodontia, Setzer e Kim (2017) afirmam que tanto os implantes dentários quanto os dentes tratados endodonticamente apresentam índices de resultados favoráveis. Não há diferença significativa no resultado entre implantes de unidade única (95\%) e dentes tratados endodonticamente (94\%) ao longo de seis anos. Os mesmos autores defendem que, em estudos de 10 anos e outros de longo prazo (> 15 anos), os dentes naturais mostraram exceder a expectativa de vida dos implantes, incluindo dentes tratados endodonticamente ou dentes comprometidos periodontalmente.

Vários autores (Setzer \& Kim 2017, Lascala 2003, Laurell et al. 1991, Yi et al. 1995, Tan et al. 2004, Lang \& Bartold 2018) demonstraram que dentes com periodonto reduzido e mobilidade dentária que foram adequadamente tratados podem ser capazes de suportar forças oclusais. A vida longa do dente depende diretamente das condições dos tecidos de suporte e um tratamento periodontal adequado (que inclui terapia não cirúrgica e cirúrgica e, mais recentemente, procedimentos regenerativos, como demonstrado por Liang, Luan, Liu, em 2020), contribuindo para o fortalecimento do periodonto de suporte. É necessário enfatizar a importância do ajuste oclusal por meio do ranger seletivo dos dentes, pois a redução da mobilidade dentária pode potencializar o efeito da terapia periodontal (Fan e Caton, 2018).

Um diagnóstico preciso das condições locais e sistêmicas e uma percepção clara da etiologia são essenciais para estabelecer um prognóstico dos dentes remanescentes. Lundgren, Rylander e Laurell (2008) apontaram que um componente decisivo para um excelente prognóstico a longo prazo no tratamento de pacientes afetados por periodontite é o controle da placa eficaz o suficiente para manter a saúde tecidos periodontais. Lindhe \& Pacey (2014) afirmam que se os pacientes com doença periodontal avançada têm seus dentes limpos e mantidos em um programa cuidadoso de controle de infecções; sua doença pode curar e desaparecer. Este é um conhecimento crucial para o clínico.

Outro elemento crucial, afirmado por Genco e Borgnakke (2013), são os fatores de risco, que desempenham um papel importante na resposta de um indivíduo à infecção periodontal. Os fatores de risco incluem suscetibilidade genética, fumo, consumo de álcool e doenças e condições não saudáveis, como diabetes mellitus, obesidade, síndrome metabólica, osteoporose e baixo teor de cálcio e vitamina D. O tabagismo representa um fator de risco significativo para complicações biológicas. Após um período de acompanhamento de 5 anos, Wennstrom et al. (2004) relataram que fumantes exibiram maior perda óssea marginal peri-implantar estatisticamente maior do que os não fumantes $(0,76 \mathrm{~mm}$ vs. $0,22 \mathrm{~mm}$, respectivamente).

Considerando o consenso de que a doença periodontal não tratada representa um risco para os implantes, devido ao estabelecimento da peri-implantite, cujo manejo terapêutico apresenta maiores dificuldades do que as encontradas na periodontite, o desenvolvimento de um tratamento periodontal adequado não representaria qualquer restrição ou oposição à colocação de um implantar. Pacientes que tiveram seus dentes extraídos e eram suscetíveis à doença periodontal ainda corriam o risco de desenvolver peri-implantite. Há evidências crescentes na literatura da alta incidência de doenças peri-implantares em pacientes com história de doença periodontal (Davarpanah 2000). Essas complicações biológicas peri-implantar graves são frequentes, e a incidência de mucosite foi relatada em cerca de $80 \%$ e a de peri-implantite entre 28 e $56 \%$ (Quirynenet al. 2002, Lindhe \& Meyle 2008). O diagnóstico, prognóstico e plano de tratamento requerem uma abordagem multidisciplinar responsável com preeminência do tratamento periodontal. A opção de preservar os dentes naturais por meio de tratamentos 
adicionais e a opção de extrair dentes comprometidos e substituí-los por implantes devem ser consideradas como opções complementares e não como concorrentes (Riccuci \& Grosso 2006). O benefício de manter até mesmo os dentes comprometidos por tratamentos endodônticos e restauradores adicionais, está hoje bem estabelecido (Naik et al. 2016). No entanto, deve ficar claro que extrações criteriosas e estratégicas não devem ser criticadas, pois podem ser muito bem indicadas e permitir a inserção de implantes nas posições necessárias (Davarpanah 2000).

Alguns autores como Lundgren, Rylander e Laurell (2008) apontam que para obter um bom prognóstico em pacientes suscetíveis à doença periodontal, alguns fatores podem ser considerados, como a gravidade da doença periodontal, demandas funcionais e estéticas do paciente cooperação e capacidade de controle de placa bacteriana, fatores de risco para falha técnica e biológica e a complexidade e custos do tratamento. Porém, o que se verifica no dia a dia das clínicas odontológicas é que o tratamento periodontal muitas vezes é ineficaz e/ou iniciado muito tarde (Nemcovsky \& Rosen 2017). Para esses autores, a Periodontia é uma disciplina que, muitas vezes, não é conduzida pelos profissionais sob os princípios da propedêutica ou da terapia baseada em evidências científicas. Além disso, Lang-Hua et al. concluíram após a aplicação de uma pesquisa por questionário a uma amostra aleatória de dentistas registrados, em uma comunidade onde o fornecimento de implantes dentários é difundido entre os Clínicos Odontológicos (PIBs), que suas atitudes não estão totalmente de acordo com o conhecimento baseado em evidências. Variações em suas atitudes existiam em relação aos fatores do dentista, treinamento e questões de experiência.

Greenstein et al. (2007) afirmam que atualmente, quando os implantes são muito valorizados, há uma tendência de subestimar o prognóstico de longo prazo de um dente natural com periodonto prejudicial. Defendendo a "racionalização", alguns profissionais entendem que manter tal dente pode danificar o local para um futuro implante ou mesmo que a inclusão desse dente em uma prótese é muito arriscada. No entanto, não existe um único parâmetro clínico confiável que possa prever a atividade da doença periodontal, a perda dentária ou a permanência dos dentes naturais em longo prazo. Esses parâmetros podem ser combinados. Na ausência de inflamação clínica e presença de periodonto saudável, mesmo que reduzido, a manutenção dos dentes naturais é vantajosa. Além disso, com o tratamento periodontal de dentes que apresentam prognóstico desfavorável, eles podem transformá-los em dentes com prognóstico favorável. Guarnieri et al. (2021) mostraram que em suas investigações a falha geral do implante foi de $10,08 \%$, e a taxa de falha do implante devido a razões biológicas foi de $9,8 \%$. A incidência de falhas de implantes em pacientes com vs sem doença periodontal recorrente foi de 83,3\% vs 16,7\% (P <05). Os resultados mostraram que em pacientes com periodontite crônica, a terapia periodontal ativa (TPA) seguida pela manutenção periodontal (PM) de longo prazo é bem-sucedida em manter a maioria dos dentes periodontais comprometidos. Nos mesmos pacientes, foi encontrada uma tendência maior para a perda do implante do que para a perda do dente.

A precaução deve ser o principal critério ao decidir sobre a extração do dente. Esta decisão depende das condições clínicas do dente e deve ser apoiada pela literatura relevante, experiência clínica, princípios éticos do profissional e desejo informado dos pacientes. Um princípio é um padrão normativo geral de conduta derivado da moralidade e das tradições na área de saúde. Cinco princípios fundamentais são discutidos nos Princípios de Ética da American Dental Association (ADA), que incluem autonomia do paciente, não maleficência, beneficência, justiça e veracidade. Oren et al. (2010) demonstraram que um total de 54 pedidos de indenização por extrações dentárias erradas foram relatados e avaliados pela Medical Consultants International de 1993 a 2004.

Zitzmann et al. (2010) apontaram que a manutenção dos dentes e a aceitação dos riscos são adequadas quando: o dente não está extensivamente doente; o dente tem um alto valor estratégico, principalmente em pacientes com contraindicações de implantes; o dente está localizado em um arco intacto e a preservação das estruturas gengivais é fundamental. Nemcosvsky, Rosen (2017) afirma que na maioria dos casos em que os dentes são extraídos por razões periodontais, a terapia com implantes exigirá grandes procedimentos de aumento ósseo. Portanto, fatores como morbidade, duração e taxas de sucesso 
devem ser avaliados cuidadosamente antes de a terapia periodontal ser descartada. Além disso, os pacientes periodontais parecem estar mais sujeitos a doenças peri-implantar e perda de implantes. Riccuci e Grosso (2006) afirmam que o desenvolvimento e o sucesso dos implantes devem ser considerados com cautela, pois há riscos de abuso quando dentes perfeitamente restauráveis são extraídos e substituídos por implantes por mera letargia e razões pecuniárias.

Os exemplos clínicos abaixo ilustram tratamentos de doença periodontal bem-sucedidos que impediram a extração de dentes e não indicavam implantes. As Figuras 1 e 2 mostram um caso de periodontite crônica generalizada em paciente do sexo feminino, tratada adequadamente, restaurando as características de normalidade clínica periodontal. Esta paciente foi instruída a extrair os dentes afetados pela doença periodontal.

Figura 1. (A) Fotografias iniciais do caso clínico e radiografias no início do estudo. Paciente do sexo feminino, 17 anos, com periodontite crônica generalizada, sangramento espontâneo, profundidade da bolsa de sondagem (PPD) entre 7 e 10 mm, extensa perda óssea e mobilidade dentária. As radiografias B e C mostram imagens abrangentes de perda óssea.

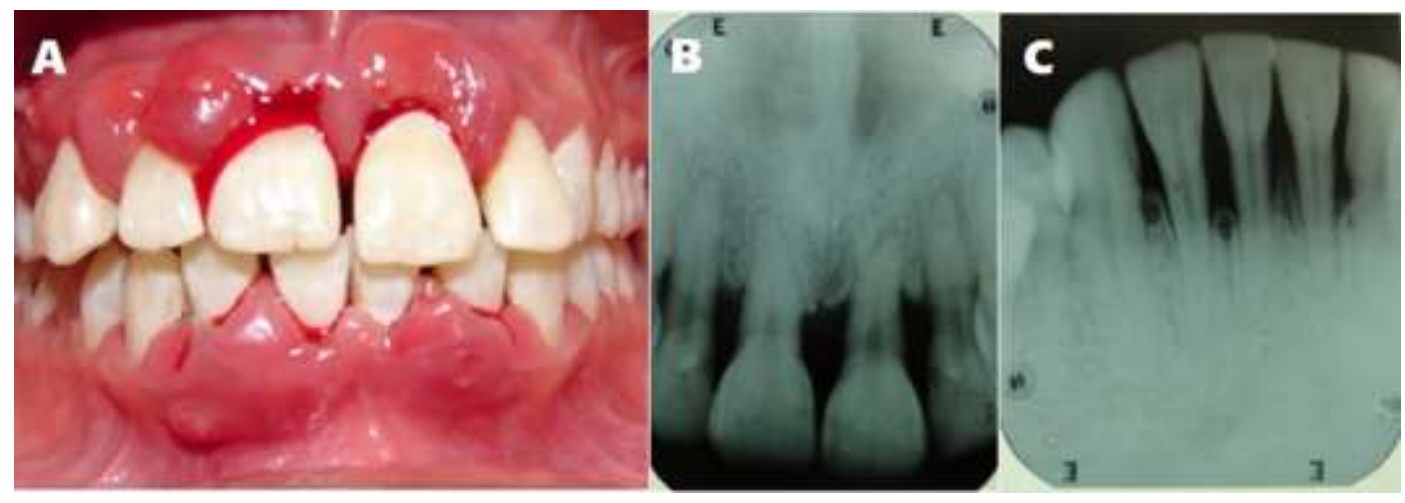

Fonte: Autores.

No primeiro caso clínico, paciente do sexo feminino, 17 anos, foi diagnosticada Diabetes Mellitus tipo 1. Na área de Periodontia, além da terapia mecânica (tratamento periodontal não cirúrgico), foram administrados Amoxicilina 500 mg e metronidazol $400 \mathrm{mg}$ por dez dias. Por fim, a paciente foi submetida a uma cirurgia de retalho periodontal (retalho de Widman modificado) (Ramfjord \& Nissle 1974), seguida de desgaste seletivo das superfícies oclusais dos dentes, para eliminar contatos prematuros e interferências oclusais.

Figura 2. Pós-operatório, 24 meses. Resolução completa do processo inflamatório, ausência de bolsas periodontais (profundidade de sondagem> $3 \mathrm{~mm}$ ) ou mobilidade dentária. Os dentes voltaram às suas posições originais após o ajuste oclusal (contato prematuro e interferências oclusais).

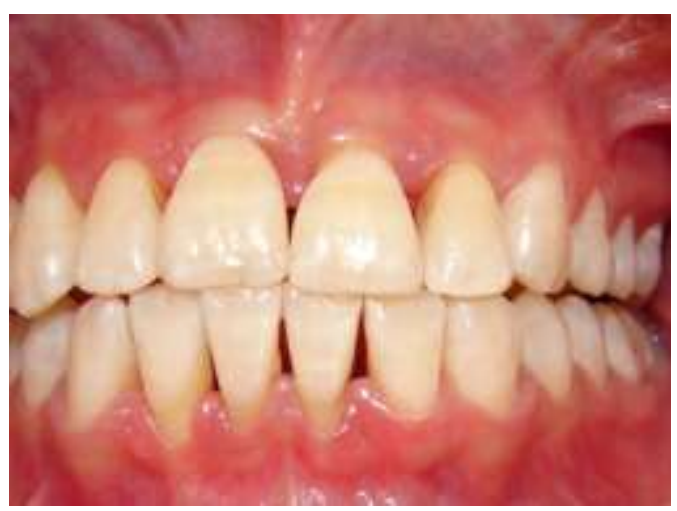

Fonte: Autores. 
No presente relato, após cirurgia periodontal, houve um reposicionamento espontâneo dos dentes migrados e restauração da estética dento-facial conforme demonstrado por Dadlani, Ramachandra, Mehta, (2013).

As Figuras 3 e 4 ilustram o caso clínico de periodontite crônica localizada em dentes anteriores inferiores.

Figura 3. Radiografia inicial do caso clínico (linha de base): periodontite crônica localizada em incisivo central inferior direito.

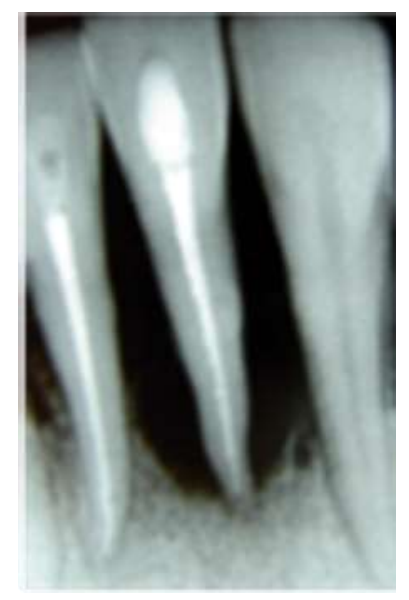

Fonte: Autores.

Observa-se extensa perda óssea radiográfica. Além disso, ao exame clínico foi detectada mobilidade dentária e bolsa periodontal. Inicialmente foi realizado um tratamento periodontal não cirúrgico (raspagem e alisamento radicular, RAR), seguido de ajuste oclusal e, em seguida, foi realizada a cirurgia de retalho de acesso periodontal (Lindhe et al. 2008).

Figura 4. (A) Radiografia pós-operatória, após 3 anos. Neoformação óssea é observada. Figura 4 (B) Aspecto clínico demonstrando resolução do processo inflamatório, ausência de mobilidade dentária e ganho do nível clínico de inserção (CAL).

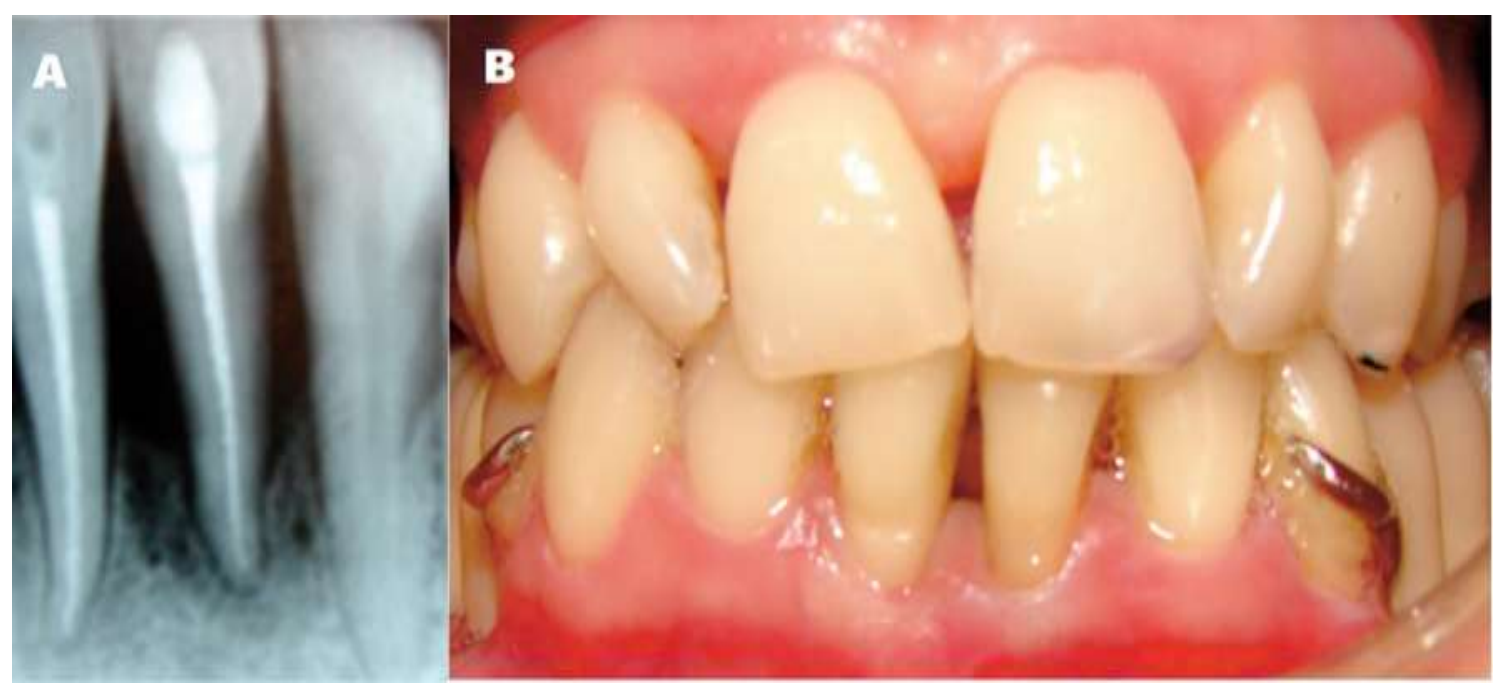

Fonte: Autores.

Após o tratamento periodontal adequado foi observada a presença de neoformação óssea na região apical, além da 
diminuição da mobilidade dentária e da bolsa periodontal.

As Figuras 5 e 6 mostram um caso de defeito de furca Classe II tratado com enxerto ósseo autógeno.

Figura 5. (A) Radiografia inicial do caso clínico (linha de base). Extensa perda óssea é observada na região da furca Classe II. (B) radiografia pós-operatória após 24 meses. Neoformação óssea é observada na região da furca e na superfície mesial do dente 46. O caso foi tratado com enxerto ósseo autógeno.

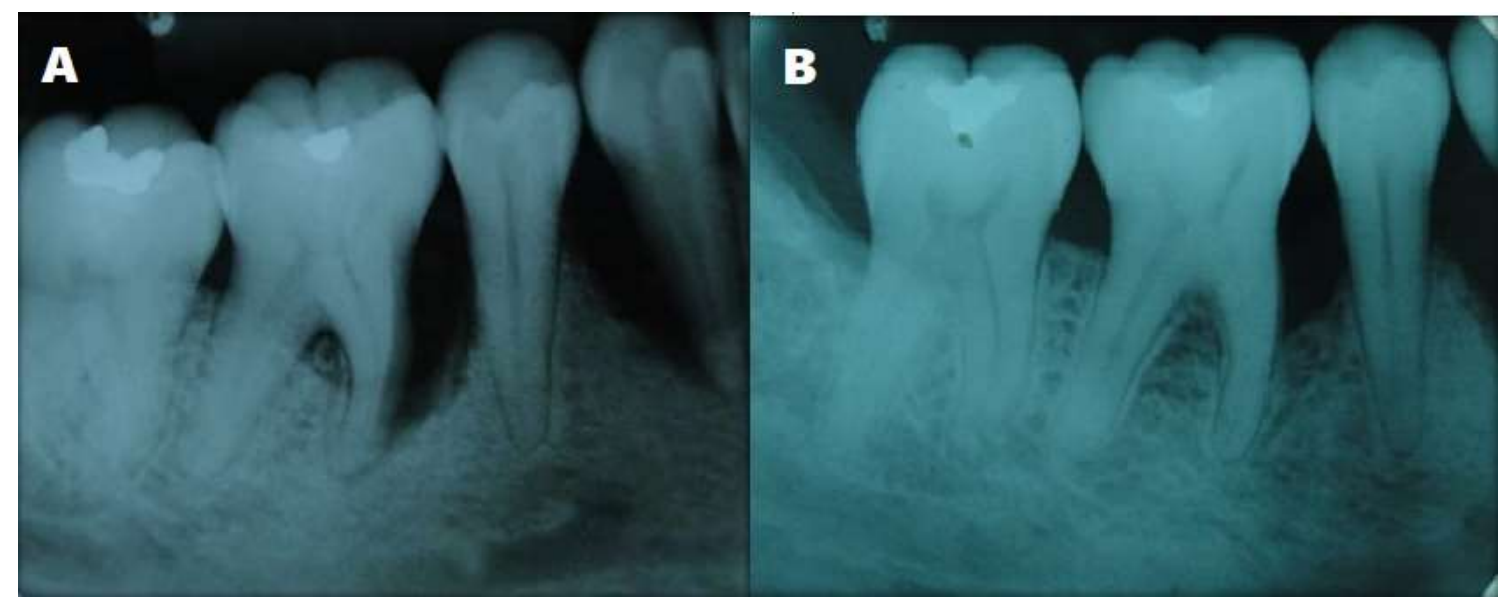

Fonte: Autores.

Observa-se a neoformação óssea e o defeito ósseo preenchido. A técnica empregada neste caso foi o coágulo ósseo (Robinson 1969, Melonig 1992).

Figura 6. Aspecto clínico pós-operatório após 24 meses da lesão na furca Classe II.

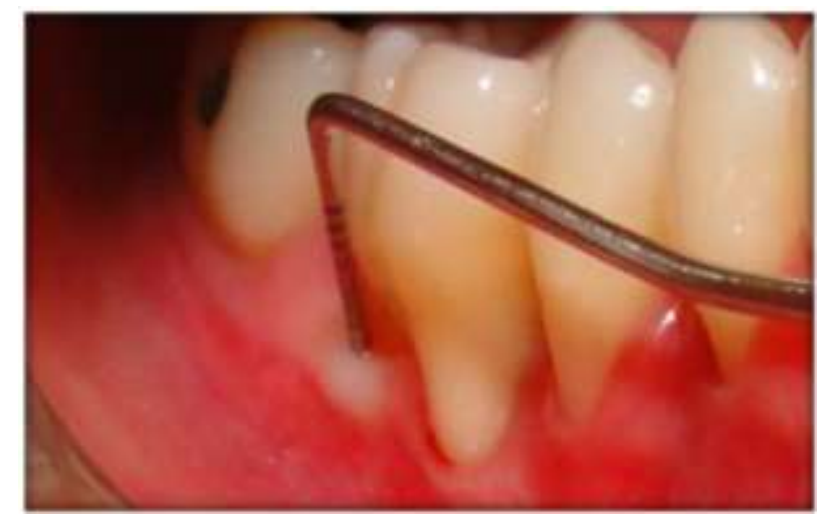

Fonte: Autores.

Observa-se resolução da inflamação e ausência de bolsas periodontais (isquemia na sondagem da margem gengival).

\section{Considerações Finais}

Um dos motivos para o aumento das exodontias é o fato de, no cotidiano da clínica odontológica, o tratamento periodontal ser visto como ineficaz e/ou iniciado tardiamente.

Com o avanço da Implantodontia, a decisão de manter os dentes naturais afetados pela doença periodontal sofreu o 
impacto de um novo prognóstico; dentes que normalmente seriam encaminhados para terapia periodontal passaram a receber indicação de exodontia, e dentes que poderiam permanecer na boca passaram a ser sumariamente substituídos por implantes.

O desenvolvimento de um tratamento periodontal adequado não significa criar restrições ou oposição aos implantes. Extrações judiciosas e estratégicas não seriam criticadas.

A decisão de extração do dente deve depender da condição clínica do dente e de um prognóstico baseado em a) na literatura relevante, b) na experiência clínica e treinamento ético do profissional, e c) no desejo informado do paciente.

Pode-se argumentar que muito mais dentes são extraídos desnecessariamente devido a diagnósticos incorretos do que desnecessariamente salvos. Um dente pode ser extraído e substituído a qualquer momento; entretanto, a extração é um tratamento definitivo e irreversível.

Futuros estudos clínicos que comparam o tratamento periodontal e implantes devem ser realizados para comprovar a eficácia clínica dos procedimentos na manutenção dentária ou de implantes.

\section{Referências}

Aida, J. Tooth Loss. In: Peres M.A., Antunes J.L.F., Watt R.G. (eds) Oral Epidemiology. Textbooks in Contemporary Dentistry. Springer, 2021.

American Dental Association. The ADA Principles of Ethics and Code of Conduct. (2018) https://www.ada.org/en/about-theada/principles-of-ethics-code-ofprofessional-conduct.

Avila, G., Galindo-Moreno, P., Soehren, S., Misch, C. E., Morelli, T. \& Wang, H. L. (2009) A Novel Decision-Making Process for Tooth Retention or Extraction. Journal of Periodontology 80(3), 476-491.

Carnevale, G., Pontoriero, R. \& di Febo, G. (1998) Long-term effects of root-resective therapy in furcation-involved molars: a 10-year longitudinal study. Journal of Clinical Periodontology 25(3), 209-214.

Dadlani, H., Ramachandra, S. S. \& Mehta, D. S. (2013) Spontaneous correction of pathologically migrated teeth with periodontal therapy alone. Journal of Indian Society of Periodontology 17(4), 531-534.

Davarpanah, M. (2000) To conserve teeth or implant: which choice of therapy? International Journal of Periodontics Restorative Dentistry 20(4), 412-422.

Doering, H. (2013) Peri-Implant Infection - A modern Phenomenon or a Long-Ignored Problem? Oral Health 1, 10.

Fan, J. \& Caton, J. G. (2018) Occlusal trauma and excessive occlusal forces: Narrative review, case definitions, and diagnostic considerations. Journal of Periodontology 89, 214-222.

Fernandez-Medina, T. \& Nanda, A. (2021) Regeneration for Implant Dentistry. In: Hosseinpour S., Walsh L.J., Moharamzadeh K. (eds) Regenerative Approaches in Dentistry.

Genco, R. J. \& Borgnakke, W.S. (2013) Risk factors for periodontal disease. Periodontology 2000, 62(1), 59-94.

Giannobile, W.V. \& Lang, N. P. (2016) Are Dental Implants a Panacea or Should We Better Strive to Save Teeth? Journal of Dental Research 95(1), 5-6.

Guarnieri, R., Di Nardo, D., Di Giorgio, G., Miccoli, G. \& Testarelli, L. (2021) Longevity of Teeth and Dental Implants in Patients Treated for Chronic Periodontitis Following Periodontal Maintenance Therapy in a Private Specialist Practice: A Retrospective Study with a 10-Year Follow-up. The International Journal of Periodontics \& Restorative Dentistry 41(1), 89-98.

Greenstein, G., Cavallaro, J. \& Tarnow, D. (2008) When to save or extract a tooth in the esthetic zone: a commentary. Compendium of Continuing Education in Dentistry 29(3), 320-321.

Greenstein, G., Greenstein, B. \& Cavallaro, J. (2007) Prerequisite for treatment planning implant dentistry: periodontal prognostication of compromised teeth. Compendium of Continuing Education in Dentistry 28(8), 436-446.

Hull, P. S., Worthington, H. V., Clerehugh, V., Tsirba, R., Davies, R.M. \& Clarkson, J. E. (1997) The reasons for tooth extraction in adults and their validation. Journal of Dentistry 25(3-4), 233-240.

Jang, H. W., Kang, J. K., Lee, K., Lee, Y. S. \& Park, P. K. (2011) A retrospective study on related factors affecting the survival rate of dental implants. Journal of Advanced Prosthodontics 3(4), 204-215.

Klinge, B., Flemming, T., Cosyn, J., De Bruyn, H., Eisner, B. M., Hultin, M., Isidor, F., Lang, N. P., Lund, B., Meyle, J., et al. (2015) The patient undergoing implant therapy: summary and consensus statements. The 4th EAO Consensus Conference 2015. Clinical Oral Implants Research 26 (Suppl 11 ), 64-67.

Lang, N. \& Bartold, M. (2018) Periodontal health. 2017 World Workshop. Journal of Clinical Periodontology 45, 9-16.

Lang, N. P., Pjetursson, B. E., Tan, K., Bragger, U. \& Zwahlen, M. (2004) A systematic review of the survival and complication rates of fixed partial dentures (FPDs) after an observation period of at least 5 years. II. Combined tooth-implant supported FPDs. Clinical Oral Implants Research 15(6), 643-653. 
Lang, N. P., Zitzmann, N. U.; Working Group 3 of the VIII European Workshop on Periodontology (2012) Clinical research in implant dentistry: evaluation of implant-supported restorations, aesthetic and patient-reported outcomes. Journal of Clinical Periodontology 39(Suppl 12), 133-138.

Lang-Hua, B. H., Lang, N. P., Lo, E. C. \& McGrath, C. P. (2013) Attitudes of general dental practitioners towards implant dentistry in an environment with widespread provision of implant therapy. Clinical Oral Implants Research 24(3), 278-284.

Lang-Hua, B. H., McGrath, C. P., Lo, E. C. \& Lang, N. P. (2014) Factors influencing treatment decision-making for maintaining or extracting compromised teeth. Clinical Oral Implants Research 25(1), 59-66.

Lascala, N. J., Jardini, M. A. \& Melo, A. B. (2003) Quais os fatores que indicam ou contraindicam a exodontia de dentes portadores de doença periodontal para a colocação de implantes osseointegrados? Periodontia e Implantodontia. Artes Médicas: São Paulo, p.465-475.

Laurell, L., Lundgren, D., Falk, H. \& Hugoson A. (1991) Long-term prognosis of extensive polyunit cantilevered fixed partial dentures. Journal of Prosthetic Dentistry 66(4), 545-552.

Lekholm, U., Gunne, J., Henry, P., Higuchi, K. Linden, J., Bergstrom, C. \& van Steenberghe, D. (1999) Survival of the Branemark Implant in partially edentulous jaws: a 10-year prospective multicenter study. International Journal of Oral \& Maxillofacial Implants 14(5), 639-645.

Levin, B. P., Rubinstein, S. \& Rose, L. F. (2015) Advanced Esthetic Management of Dental Implants: Surgical and Restorative Considerations to Improve Outcomes. Journal of Esthetic and Restorative Dentistry 27(4), 224-230.

Liang, Y., Luan, X. \& Liu, X. (2020) Recent advances in periodontal regeneration: a biomaterial perspective. Bioactive Materials 5(2), $297-308$.

Lindhe, J., Lang, J. \& Karring, N. P. (2008) Clinical periodontology and implant dentistry, 5th edition. Wiley-Blackwell. UK.

Lindhe, J. (2007) The bacterial plaque hunter. Revista Dental Press de Periodontia e Implantologia 1, 14-29.

Lindhe, J. \& Meyle, J. (2008) Peri-implant diseases: Consensus Report of the Sixth European Workshop on Periodontology. Journal of Clinical Periodontology 35 (8 Suppl), 282-285.

Lindhe J. \& Pacey, L. (2014) There is an overuse of implants in the world and an underuse of teeth as targets for treatment. British Dental Journal 217(8), 396-397.

Lundgren, D., Rylander, H. \& Laurell, L. (2008). To save or to extract, that is the question. Natural teeth or dental implants in periodontitis susceptible patients: clinical decision-making and treatment strategies exemplified with patient case presentations. Periodontology 2000 47, 27-50.

Melonig, J. (1992) Autogenous and Allogeneic Bone Grafts in Periodontal Therapy. Critical Reviews in Oral Biology \& Medicine 3(4), 333-352.

Naik, S. P., Nivedan, K. S., Pinto, B., Shobha, R., Dutt, A. S. \& Rahul, N. (2016) Ethical Issues and Challenges in Dentistry. International Journal of Preventive and Clinical Dental Research 3(4), 285-287.

Nemcovsky, C. \& Rosen, E. (2017) Biological complications in implant-supported oral rehabilitation: as the pendulum swings back towards endodontics and tooth preservation. Evidence-Based Endodontics 2, 1-8.

Nyman, S. \& Lindhe, J. (1979) A longitudinal study of combined periodontal and prosthetic treatment of patients with advanced periodontal disease. Journal of Periodontology 50(4), 163-169.

Ong, G. (1998) Periodontal diseases and tooth loss. International Dentistry Journal 48(3 Suppl 1), 233-228.

Oren, P., Navot, G, Tali, H.S. \& Shlomo, T. (2010) Wrong tooth extraction: Root cause analysis. Quintessence International 41(10), 869-872.

Patel, R. M. (2020) Dental Implants for Patients with Periodontitis. Primary Dental Journal. 8(4), 54-61.

Pereira, A. S., Shitsuka, D. M., Parreira, F. J. \& Shitsuka, R. (2018) Metodologia da Pesquisa Científica. 1. ed. - Santa Maria, RS: UFSM, NTE, 119 p.

Pjetursson, B. E., Tan, K., Lang, N. P., Bragger, U., Egger, M. \& Zwahlen, M. (2004). A systematic review of the survival and complication rates of fixed partial dentures (FPDs) after an observation period of at least 5 years. I. Implant-supported FPDs. Clinical Oral Implants Research 15(6), 625-642.

Quirynen, M., De Soete, M. \& Van Steenberghe, D. (2002) Infectious risks for oral implants: a review of the literature. Clinical Oral Implant Research 13(1), $1-19$.

Ramfjord, S. P. \& Nissle, R. R. (1974) The modified Widman flap. Journal of Periodontology 45(8), 601-607.

Riccuci, S. \& Grosso, A. (2006) The compromised tooth: conservative treatment or extraction? Endodontic Topics 13, 108-122.

Robinson, R. E. (1969) Osseous Coagulum for Bone Induction. Journal of Periodontology 40(9), 503-510.

Salvi, G. E., Mischler, D. C., Schmidlin, K., Matuliene, G., Pjetursson, B. E., Brägger, U. \& Lang, N. P. (2014) Risk factors associated with the longevity of multi-rooted teeth. Long-term outcomes after active and supportive periodontal therapy. Journal of Clinical Periodontology 41(7), 701-707.

Sanz, M., Herrera, D., Kebschull, M., Chapple, I., Jepsen, S., Beglundh, T., Sculean, A., \& Tonetti, M. S.; EFP Workshop Participants and Methodological Consultants. (2020) Treatment of stage I-III periodontitis-The EFP S3 level clinical practice guideline. Journal of Clinical Periodontology 47 Suppl 22(Suppl 22), 4-60.

Schimmel, M., Srinivasan, M., McKenna, G. \& Müller, F. (2018) Effect of advanced age and/or systemic medical conditions on dental implant survival: A systematic review and meta-analysis. Clinical Oral Implants Research 29, 311-331 
Setzer, F. \& Kim, S. (2017) Preserving the Natural Tooth Versus Extraction and Implant Placement: An Evidence-Based Approach. In: Rosen, E., Nemcovsky, C. \& Tsesis I. Refuat Hapeh Vehashinayim (1993) 2010 27, 37-46.

Tallarico, M., Caneva, M., Baldini, N., Gatti, F., Duvina, M., Billi, M., Iannello, G., Piacentini, G., Meloni, S. M. \& Cicciù M (2018) Patient-centered rehabilitation of single, partial, and complete edentulism with cemented- or screw-retained fixed dental prosthesis: The First Osstem Advanced Dental Implant Research and Education Center Consensus Conference 2017. European Journal of Dentistry 12(4), 617-626.

Tan, K., Pjetursson, B. E., Lang, N. P. \& Chan, E. S. Y. (2004) A systematic review of the survival and complication rates of fixed partial dentures (FPDs) after an observation period of at least 5 years. III. Conventional FPDs. Clinical Oral Implants Research 15(6), $654-666$.

Wennström, J. L., Ekestubbe, A., Gröndahl, K., Karlsson, S. \& Lindhe, J. (2004) Oral rehabilitation with implant-supported fixed partial dentures in periodontitis-susceptible subjects. A 5-year prospective study. Journal of Clinical Periodontology 31(9), 713-724.

Yi, S. W., Ericsson, I., Carlsson, G. \& Wennstrom, J. L. (1995) Long-term follow-up of cross-arch fixed partial dentures in patients with advanced periodontal destruction. Acta Odontologica Scandinavica 53(4), 242-248.

Zitzmann, N., Krastl, G., Hecker, H., Walter, C., Waltimo, T. \& Weiger, R. (2010) Strategic considerations in treatment planning: Deciding when to treat, extract, or replace a questionable tooth. Journal of Prosthetic Dentistry 104(2), 80-91. 